\title{
Effect of Alcohol Consumption during Antiretroviral Therapy on HIV-1 Replication: Role of Cytochrome P3A4 Enzyme
}

\author{
Srijita Mondal \\ Centre for Mathematical Biology and Ecology \\ Department of Mathematics \\ Jadavpur University, Kolkata - 700032, India \\ Priyanka Ghosh \\ Centre for Mathematical Biology and Ecology \\ Department of Mathematics \\ Jadavpur University, Kolkata - 700032, India \\ Dibyendu Biswas \\ Department of Mathematics \\ City College of Commerce and Business Administration \\ 13, Surya Sen Street, Kolkata-700012, India \\ Priti Kumar Roy \\ Centre for Mathematical Biology and Ecology \\ Department of Mathematics \\ Jadavpur University, Kolkata - 700032, India \\ Corresponding author: pritiju@ gmail.com
}

(Received July 18, 2018; Accepted April 19, 2019)

\begin{abstract}
Alcohol consumption is prevalent in HIV/AIDS infected patients. It possesses serious effects on protease inhibitors (PIs), which are used as an antiviral drug. While taking PIs, the secretion of Cytochrome P3A4 (CYP3A4) enzymes occurs from the liver and it metabolizes the drug to CYP3A4-PI complex. Alcohol consumption increases the rate of metabolism of PIs. In this research article, we have formulated a set of nonlinear differential equations based on the enzymatic activity of CYP3A4 for alcoholic HIV infected patients. Here, we have analytically compared the dynamics of PIs metabolism between alcoholic and non-alcoholic HIV infected patients and also investigated how the infection is being accelerated by enhancing viral load due to alcohol consumption. Finally, our analytical results are verified by numerical findings.
\end{abstract}

Keywords- HIV/AIDS, Viral load, Alcohol consumption, CYP3A4 enzyme, Protease inhibitor.

\section{Introduction}

HIV-1 infection is a global problem with 36.9 million people affected in the world at the end of 2017. The African region remains most severely affected with accounting for nearly two-thirds of the people living with HIV worldwide (Kumar et al., 2012). There were approximately 1.8 million people becoming newly infected in 2017 globally. People with HIV infection have to take a combination of anti-AIDS drugs on a daily basis to stay healthy.

There are about 26 FDA approved anti-AIDS drugs currently available acting on different stages of the HIV life-cycle (Das and Arnold, 2013); most fall into 4 classes: (i) Entry/Fusion Inhibitors 
International Journal of Mathematical, Engineering and Management Sciences

Vol. 4, No. 4, 922-935, 2019

https://dx.doi.org/10.33889/IJMEMS.2019.4.4-073

(ii) Reverse Transcriptase Inhibitors (Nucleoside/Nucleotide Reverse Transcriptase Inhibitors, i.e., NRTI and Non-Nucleoside Reverse Transcriptase inhibitors, i.e., NNRTI) (iii) Integrase Inhibitors and (iv) Protease Inhibitors, i.e., PI. The most extensive treatment approach for acutely infected HIV patients is highly active antiretroviral therapy (HAART), which typically includes 2 NRTIs along with 1NNRTI and PI.

The prevalence of alcohol abuse is approximately three times higher in HIV-infected individuals than in the general population and is associated with adverse health effects (Purohit et al., 2011; Chander, 2011; Gonzalez et al., 2011; Kumar et al., 2012). Heavy alcohol consumption and alcoholism can cause liver and pancreatic damage. Also, alcohol increases the progress rate of AIDS and neuroAIDS (Chander et al., 2009; Norman and Kumar, 2006). Although HIV-infected patients should avoid alcohol especially while on HAART treatment, patient compliance is limited (Miguez et al., 2003; Hirsch et al., 2008;). Chronic alcohol consumption during HAART decreases patient adherence to HAART as well as increases neuronal toxicity (Witteveen and van Ameijden, 2002; Chander et al., 2006; Hirsch et al., 2008; Hendershot et al., 2009; Ferrari and Levine, 2010). The enzyme Cytochrome P450 3A4 (CYP3A4) plays an important role in interactions between antiretrovirals (ART) and alcohol.

Cytochrome P450 3A4 (CYP3A4) is the major metabolic enzyme in the human liver, which is responsible to metabolize about $50 \%$ of the therapeutic drugs including non-nucleoside reverse transcriptase inhibitors (NNRTIs) and protease inhibitors (PIs) (Midde et al., 2016; Kumar and Kumar, 2011). Inhibition or inactivation of CYP3A4 can cause mild to severe drug-drug interactions resulting in unanticipated adverse reactions or therapeutic failures (Kumar and Kumar, 2011; Pal and Mitra, 2006; Walubo, 2007). NNRTIs and PIs act as either inducers or inhibitors of CYP3A4 while all PIs are inhibitors of CYP3A4 (Kumar et al., 2012). Ritonavir, a protease inhibitor which is an extremely potent inhibitor of CYP3A4 is present in most of the HAART regimens. It is generally used as a booster to increase the plasma concentrations and the half-life of other ARTs because of its strong binding affinity to CYP3A4 and inhibition of CYP3A4 (Kumar and Kumar, 2011). Thus, ritonavir increases the bioavailability of NNRTIs and PIs by decreasing their metabolism by CYP3A4 (Acosta, 2002; Xu and Desai, 2009; Kumar et al., 2012). But in this case, alcohol consumption may alter the rate of metabolism of PIs by changing CYP3A4-PIs interactions.

Alcohol interacts with PIs, through CYP3A4 induction leading to altered drug metabolism and toxicity in the liver (Flexner et al., 2001; Kumar et al., 2012). Since Ethanol has greater binding affinity to enzyme CYP3A4 than PIs, it first binds with the enzyme forming CYP3A4-Ethanol complex. Thus Ethanol alters the inhibition of CYP3A4 by PIs which may lead to relatively faster metabolism of PIs. For this, the effectiveness of the PIs, as well as, NNRTIs decreases. Thus, individuals who consume ethanol and take PI drugs are at high risk of deleterious ethanol PIs interaction.

There are ample mathematical models available in literature considering HIV/AIDS transmission those are being implemented either in cellular or in social population level. In 2015, Roy et al. (2015) determined the media impact on the HIV outbreak and suggested that infection level reduces the effective contacts between human and media. Also, it has been shown the impact of an awareness campaign to combat Leishmaniasis (Biswas et al., 2016), and the methodology of satellite data utilization in Multi-Modeling Approach for socio-ecological risks (Kostyuchenko, 2018). A handful of mathematical models have examined various treatment strategies and the 
International Journal of Mathematical, Engineering and Management Sciences

Vol. 4, No. 4, 922-935, 2019

https://dx.doi.org/10.33889/IJMEMS.2019.4.4-073

effects of different drug therapies. The effect of perfect adherence to antiretroviral therapy has been studied using impulsive differential equations (Smith and Wahl, 2004; Smith and Wahl, 2005; Lou and Smith, 2011; Smith?, 2008; Song et al., 2011). Several mathematical models have been developed to describe the interaction of the human immune system with HIV, the decline in CD4+ T cell count and the effects of different drug therapies (Chatterjee and Roy, 2012). Recently, Chowdhury and Roy (2016) examined the effect of combination therapy on viral dynamics using Enfuvirtide and PIs. Also, several studies have shown the alcohol consumption effects for HIV-infected patients while they are on HAART. They also describe the role of CYP450 enzymes in the metabolism of drugs used in HAART (i.e., Protease Inhibitors (PI) and non-nucleoside reverse transcriptase inhibitors (NNRTI)) (Kumar and Kumar, 2011; Kumar et al., 2012). Even Ghosh et al. has done some mathematical works by formulating enzyme kinetics models on alcoholism (Ghosh and Roy, 2018; Ghosh et al., 2018). Although there is a lot of information on experimental works involving the effects of ethanol on PIs, there is a need to establish mathematical models to improve our understanding of these processes. Thus, in this research work, we have formulated a mathematical model describing the CYP3A4-ethanol interactions involved in order to alter drug metabolism. We start by making schematic themes where we show how consumption of alcohol inhibits CYP3A4-PI interaction. The model is studied both analytically and numerically based on the biological and chemical interactions of CYP3A4-PI-Ethanol complexes.

The research article has been arranged as follows: Section (2) describes the model formulation followed by the analytical part in section (3). Section (4) deals with the numerical experimentation and section (5) contains the conclusion.

\section{Formulation of Mathematical Model}

For the HIV infected patients, Protease Inhibitors $(I)$ are metabolized into the CYP3A4-PI complex $(E I)$ by the liver secreted cytochrome P3A4 (CYP3A4) enzyme $(E)$ and little portion of the complex $(E I)$ reverts. For this reaction, the forward and the backward rate constants are $\mathrm{k}_{1}$ and $\mathrm{k}_{-1}$ respectively. Since, alcohol $(S)$ has higher affinity towards the cytochrome P3A4 (CYP3A4) enzyme (E) than PIs (I), alcohol binds with the single active sited enzyme $(E)$ for an alcoholic HIV infected patient and forms enzyme-alcohol complex (ES). A little portion of this complex $(E S)$ reverts and remaining portion of it breaks down into the alcohol $\left(S^{\prime}\right)$ and the enzyme $\left(E_{S}\right)$ again, slight changing their internal properties. The released enzyme $\left(E_{S}\right)$ becomes more strong than previous one, which actually helps to bind and metabolize the PIs $(I)$ more effectively. For this, the utilization of the drug PIs $(I)$ decreases and metabolism of it increases, which is harmful for an HIV infected patient. However, the enzyme $\left(E_{S}\right)$ and PI $(I)$ reacting with each other form intermediate complex $\left(E_{S} I\right)$, a little portion of which reverts and remaining portion is transformed into another complex $\left(E_{S} I S^{\prime}\right)$ binding with the changed alcohol $\left(S^{\prime}\right)$. Finally, the last complex $\left(E_{S} I S^{\prime}\right)$ breaks down into a product $(P)$ with unrecognised property and the enzyme $\left(E_{S}\right)$ as well as slight portion of it $\left(E_{S} I S^{\prime}\right)$ reverts. The forward and the backward rate constants for this alcohol induced PIs metabolism system are $k_{2}, k_{3}, k_{4}, k_{5}, k_{6}$ and $k_{-2}, k_{-4}, k_{-5}$ respectively. The above facts are represented in the schematic diagram as in Figure 1. 
International Journal of Mathematical, Engineering and Management Sciences

Vol. 4, No. 4, 922-935, 2019

https://dx.doi.org/10.33889/IJMEMS.2019.4.4-073

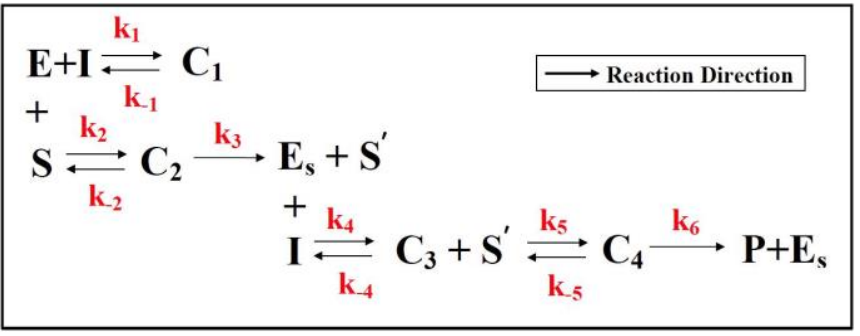

Figure 1. The schematic diagram of competitive inhibition reaction

To simplify the above system and avoid the complexity of the analytical as well as numerical studies we can imagine a zoom out image of the whole system.

We skip the further break down of $C_{2}$ and therefore the concentrations of $E_{S}$ and $S^{\prime}$ are automatically omitted from the second horizontal line of the reaction chain. Carrying the consequence of the second row, $C_{4}$ is also deleted with $S^{\prime}$ from the third row as $C_{4}$ is nothing other than a complex from $S^{\prime}$ and $C_{3}$ reaction. Hence, here we neglect two further steps of reaction in two different horizontal lines. In fact, if we think more realistically those steps are truly negligible for the rapidity of the reaction. Except those ignored parts, the schematic diagram is same as above and the notations carry their usual meaning. Furthermore, there are some other notations seen in the inland mathematical model. For instance, $\lambda$ is the secretion rate of enzyme $E$ and $\mu_{1}, \mu_{2}, \mu_{3}$ and $\mu_{4}$ are the natural dissociation or clearance rates of $E, I, S$ and $P$ respectively. In addition, $\overline{\mathrm{I}}$ and $\overline{\mathrm{S}}$ are the rates of required concentration for $I$ and $S$, those absorb in the mentioned enzymatic reaction. Now, the modified schematic diagram is as in Figure 2.

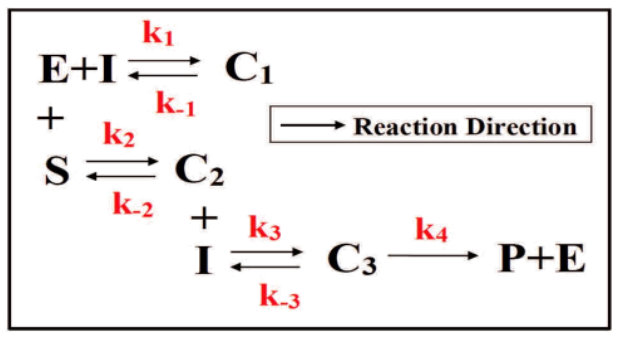

Figure 2. The schematic diagram of competitive inhibition reaction

Following the Law of Mass Action, the set of nonlinear differential equations for the above enzymatic reaction is as follows: 
International Journal of Mathematical, Engineering and Management Sciences

Vol. 4, No. 4, 922-935, 2019

https://dx.doi.org/10.33889/IJMEMS.2019.4.4-073

$$
\begin{aligned}
& \frac{d E}{d t}=\lambda-k_{1} E I+k_{-1} C_{1}-k_{2} E S+k_{-2} C_{2}+k_{4} C_{3}-\mu_{1} E \\
& \frac{d I}{d t}=\bar{I}-k_{1} E I+k_{-1} C_{1}-k_{3} C_{2} I+k_{-3} C_{3}-\mu_{2} I \\
& \frac{d C_{1}}{d t}=k_{1} E I-k_{-1} C_{1} \\
& \frac{d S}{d t}=\bar{S}-k_{2} E S+k_{-2} C_{2}-\mu_{3} S \\
& \frac{d C_{2}}{d t}=k_{2} E S-k_{-2} C_{2}-k_{3} C_{2} I+k_{-3} C_{3} \\
& \frac{d C_{3}}{d t}=k_{3} C_{2} I-k_{-3} C_{3}-k_{4} C_{3} \\
& \frac{d P}{d t}=k_{4} C_{3}-\mu_{4} P
\end{aligned}
$$

with the initial conditions $E(0)=E_{0}, I(0)=I_{0}, C_{1}(0)=C_{10}, S(0)=S_{0}, C_{2}(0)=0, C_{3}(0)=$ 0 and $P(0)=0$.

\section{Model Property}

\subsection{Non-Negativity}

In this section, we want to show the non-negativity of the solutions of the system (1) using the following theorem.

Theorem 3.1. Each solution of the system (1) with initial conditions remains non-negative for all $t \geq 0$.

\section{Proof.}

From the first equation of system (1), we can write,

$$
\begin{aligned}
& \frac{d E}{d t}=\lambda-k_{1} E I+k_{-1} C_{1}-k_{2} E S+k_{-2} C_{2}+k_{4} C_{3}-\mu_{1} E \\
& >-k_{1} E I-k_{2} E S-\mu_{1} E \quad \forall t \in\left[0, t_{1}\right] .
\end{aligned}
$$

Hence,

$E(t)>E(0) e^{-\int_{0}^{t}\left(k_{1} I(T)+k_{2} S(T)+\mu_{1}\right) d T}$,

as $t \in\left[0, t_{1}\right]$ and as long as

$$
\int_{0}^{t} I(T) d T<+\infty \text { and } \int_{0}^{t} S(T) d T<+\infty .
$$

Again from the second equation, we have

$$
\begin{aligned}
\frac{d I}{d t}=\bar{I}-k_{1} E I & +k_{-1} C_{1}-k_{3} C_{2} I+k_{-3} C_{3}-\mu_{2} I \\
> & -k_{1} E I-k_{3} C_{2} I-\mu_{2} I \quad \forall t \in\left[0, t_{1}\right] .
\end{aligned}
$$


International Journal of Mathematical, Engineering and Management Sciences

Vol. 4, No. 4, 922-935, 2019

https://dx.doi.org/10.33889/IJMEMS.2019.4.4-073

which implies,

$I(t)>I(0) e^{-\int_{0}^{t}\left(k_{1} E(T)+k_{3} C_{2}(T)+\mu_{2}\right) d T}$,

as $t \in\left[0, t_{1}\right]$ and as long as

$$
\int_{0}^{t} E(T) d T<+\infty \quad \text { and } \quad \int_{0}^{t} C_{2}(T) d T<+\infty
$$

In a similar way we can show the non-negativity of $S$ and $C_{1}$ in $\left[0, t_{1}\right]$.

Now, we prove the non-negativity of $C_{2}, C_{3}$ and $P$.

Here,

$$
\begin{array}{r}
\frac{d C_{2}}{d t}=k_{2} E S-k_{-2} C_{2}-k_{3} C_{2} I+k_{-3} C_{3} \\
>-k_{-2} C_{2}-k_{3} C_{2} I .
\end{array}
$$

But the initial value of $C_{2}$ is zero. So, we can write,

$\frac{d C_{2}}{d t} \geq 0$

Implies

$C_{2} \geq 0$.

In similar manner we can also show the non-negativity of $C_{3}$ and $P$.

Thus, we prove the non-negativity of the solutions of the system (1) with the initial conditions.

\subsection{Boundedness}

In this section, it is shown that the solutions of the system (1) are bounded, using the following theorem.

Theorem 3.2. Every solution of the system (1) with initial conditions, starts in $R_{+}^{7}$, is uniformly bounded.

Proof: $\lambda, \overline{\mathrm{I}}$ and $\overline{\mathrm{S}}$ are the entering rate of $E, I$ and $S$ respectively for the proposed enzymatic reaction. It is obvious that the concentration of $E, I$ and $S$ always lie within their entering concentration. Therefore, we can write $E \leq \lambda, I \leq \bar{I}, S \leq \bar{S}$ for any time $t$.

Now, from the third equation of the system (1), we can write

$$
\frac{d C_{1}}{d t}=k_{1} E I-k_{-1} C_{1} \leq k_{1} \lambda \bar{I}-k_{-1} C_{1}
$$

Solving the above inequality, we get

$$
C_{1} \leq \frac{k_{1} \lambda \bar{I}}{k_{-1}}+C_{1}(0) e^{-k_{-1} t},
$$


International Journal of Mathematical, Engineering and Management Sciences

Vol. 4, No. 4, 922-935, 2019

https://dx.doi.org/10.33889/IJMEMS.2019.4.4-073

for long time interval, we obtain

$C_{1}(t) \leq \frac{k_{1} \lambda \bar{I}}{k_{-1}}$, the maximum value of CYP3A4-PI.

Now, we add fifth and sixth equations of our model

$\frac{d\left(C_{2}+C_{3}\right)}{d t} \leq k_{2} \lambda \bar{S}-\xi\left(C_{2}+C_{3}\right)$, where $\xi=\min \left\{k_{-2}, k_{4}\right\}$

Solving the above inequality, we get

$\left(C_{2}+C_{3}\right)(t) \leq \frac{k_{2} \lambda \bar{s}}{\xi}+\left(C_{2}+C_{3}\right)(0) e^{-\xi t}$,

for long time interval, we get

$\left(C_{2}+C_{3}\right)(t) \leq \frac{k_{2} \lambda \bar{s}}{\xi}$

the maximum value of CYP3A4-Ethanol complex and CYP3A4-PI-Ethanol complex.

Similarly, using the maximum value of $C_{3}$, we get from last equation,

$\frac{d P}{d t} \leq \frac{k_{2} k_{4} \lambda \bar{S}}{\xi}$

Solving the above inequality, we get the threshold value of product as follows,

$P(t) \leq \frac{k_{2} k_{4} \lambda \bar{S}}{\xi \mu_{4}}$

Therefore, all solutions of the system (1) are bounded.

\subsection{Equilibrium Analysis}

In this section we analyse the stability of the endemic equilibrium of the system (1). The endemic equilibrium of the system (1) will be in the form $E\left(E^{*}, I^{*}, S^{*}, C_{1}{ }^{*}, C_{2}{ }^{*}, C_{3}{ }^{*}, P^{*}\right)$, where, $E^{*}=$ $\frac{\lambda}{\mu_{1}}, I^{*}=\frac{\bar{I}-k_{4} C_{3}}{\mu_{2}}, S^{*}=\frac{\bar{s}-k_{4} C_{3}}{\mu_{3}}, C_{1}{ }^{*}=\frac{\lambda k_{1}\left(\bar{I}-k_{4} C_{3}\right)}{k_{-1} \mu_{1} \mu_{2}}, C_{2}{ }^{*}=\frac{\lambda k_{2} \bar{S}-\lambda k_{2} k_{4} C_{3}-\mu_{1} \mu_{3} k_{4} C_{3}}{k_{-2} \mu_{1} \mu_{3}}, P^{*}=\frac{k_{4} C_{3}}{\mu_{4}} \quad$ and the value of $C_{3}{ }^{*}$ will be obtained from the equation:

$$
\begin{gathered}
\left(\lambda k_{2} k_{3} k_{4}^{2}+\mu_{1} \mu_{3} k_{3} k_{4}^{2}\right) C_{3}^{* 2}-\left(\lambda k_{2} k_{3} k_{4} \bar{S}+\lambda k_{2} k_{3} k_{4} \bar{I}+\mu_{1} \mu_{3} k_{3} k_{4} \bar{I}+k_{2} k_{3} \mu_{1} \mu_{2} \mu_{3}\right. \\
\left.+k_{2} k_{4} \mu_{1} \mu_{2} \mu_{3}\right) C_{3}{ }^{*}+\lambda k_{2} k_{3} \bar{S} \bar{I}=0 .
\end{gathered}
$$

The Jacobian matrix at the equilibrium point is: 
International Journal of Mathematical, Engineering and Management Sciences

Vol. 4, No. 4, 922-935, 2019

https://dx.doi.org/10.33889/IJMEMS.2019.4.4-073

$$
J=\left(\begin{array}{ccccccc}
-k_{1} I^{*}-k_{2} S^{*}-\mu_{1} & -k_{1} E^{*} & -k_{2} E^{*} & k_{-1} & k_{-2} & k_{4} & 0 \\
-k_{1} I^{*} & -k_{1} E^{*}-k_{3} C_{2}{ }^{*}-\mu_{2} & 0 & k_{-1} & -k_{3} I^{*} & k_{-3} & 0 \\
-k_{2} S^{*} & 0 & -k_{2} E^{*}-\mu_{3} & 0 & k_{-2} & 0 & 0 \\
k_{1} I^{*} & k_{1} E^{*} & 0 & -k_{-1} & 0 & 0 & 0 \\
k_{2} S^{*} & -k_{3} C_{2}{ }^{*} & k_{2} E^{*} & 0 & -k_{-2}-k_{3} I^{*} & k_{-3} & 0 \\
0 & k_{3} C_{2}{ }^{*} & 0 & 0 & k_{3} I^{*} & -k_{-3}-k_{4} & 0 \\
0 & 0 & 0 & 0 & 0 & k_{4} & -\mu_{4}
\end{array}\right)
$$

Let, elements of the above matrix $J$ are of the form $a_{i j}$ where $i, j \in\{1,2, \ldots, 7\}$. Now, the characteristic polynomial of the above matrix $J$ is,

$\operatorname{det}\left(J-u I_{7}\right)=\left(-\mu_{4}-u\right)\left(u^{6}+A u^{5}+B u^{4}+C u^{3}+D u^{2}+E u+F\right)$.

where the values of the coefficients $A, B, C, D, E, F$ are as follows:

$$
\begin{aligned}
& A=-\sum a_{i i} \\
& B=\sum a_{i i} a_{j j}-\sum a_{i j} a_{j i} \\
& C=-\sum a_{i i} a_{j j} a_{k k}+\sum a_{i j} a_{j i} a_{k k}-\sum a_{i j} a_{j k} a_{k i} \\
& D=\sum a_{i i} a_{j j} a_{k k} a_{l l}+\sum a_{i j} a_{j i} a_{k i} a_{l l}+\sum a_{i j} a_{j i} a_{k l} a_{l k}-\sum a_{i j} a_{j i} a_{k k} a_{l l} \\
& -\sum a_{i j} a_{j k} a_{k l} a_{l i} \\
& E=-\sum a_{i i} a_{j j} a_{k k} a_{l l} a_{m m}+\sum a_{i j} a_{j i} a_{k k} a_{l l} a_{m m}-\sum a_{i j} a_{j k} a_{k l} a_{l i} a_{m m} \\
& -\sum a_{i j} a_{j k} a_{k i} a_{l l} a_{m m}-\sum a_{i j} a_{j i} a_{k l} a_{l k} a_{m m}+\sum a_{i j} a_{j k} a_{k l} a_{l m} a_{m i} \\
& F=\sum a_{i i} a_{j j} a_{k k} a_{l l} a_{m m} a_{n n}+\sum a_{i j} a_{j k} a_{k l} a_{l m} a_{m i} a_{n n}+\sum a_{i j} a_{j i} a_{k l} a_{l k} a_{m m} a_{n n} \\
& +\sum a_{i j} a_{j k} a_{k i} a_{l m} a_{m n} a_{n l}-\sum a_{i j} a_{j i} a_{k k} a_{l l} a_{m m} a_{n n}-\sum a_{i j} a_{j i} a_{k l} a_{l m} a_{m k} a_{n n}
\end{aligned}
$$

Here $A, B, C, D, E, F$ follows a rule: $i \neq j \neq k \neq m \neq n$. If $i=1$, then $j$ can go to 2 or 3 or 4 or 5 or 6 . Following same rule for $k, l, m, n$. . Similarly, 2 can go to 1 or 4 or 5 or 6,3 can go to 1 or 5,4 can go to 1 or 2,5 can go to 1 or 2 or 3 or 6 , and 6 can go to 2 or 5 .

Let $M_{1}=(A), M_{2}=\left(\begin{array}{cc}A & 1 \\ 0 & B\end{array}\right), M_{3}=\left(\begin{array}{lll}A & 1 & 0 \\ C & B & A \\ 0 & 0 & C\end{array}\right), M_{4}=\left(\begin{array}{cccc}A & 1 & 0 & 0 \\ C & B & A & 1 \\ 0 & D & C & B \\ 0 & 0 & 0 & D\end{array}\right)$, 
International Journal of Mathematical, Engineering and Management Sciences

Vol. 4, No. 4, 922-935, 2019

https://dx.doi.org/10.33889/IJMEMS.2019.4.4-073

$$
M_{5}=\left(\begin{array}{ccccc}
A & 1 & 0 & 0 & 0 \\
C & B & A & 1 & 0 \\
E & D & C & B & A \\
0 & 0 & E & D & C \\
0 & 0 & 0 & 0 & E
\end{array}\right) \text { and } M_{6}=\left(\begin{array}{cccccc}
A & 1 & 0 & 0 & 0 & 0 \\
C & B & A & 1 & 0 & 0 \\
F & E & D & C & B & A \\
0 & F & E & D & C & B \\
0 & 0 & 0 & F & E & D \\
0 & 0 & 0 & 0 & 0 & F
\end{array}\right)
$$

Lemma: All roots of the characteristic equation are negative or negative real part if the determinants of all the Hurwitz matrices are positive, i.e., $\operatorname{det}\left(M_{j}\right)>0, j \in\{1,2, \ldots, 6\}$. Thus from the Routh-Hurwitz criterion the system is asymptotically stable if $\operatorname{det}\left(M_{j}\right)>0, j \in$ $\{1,2, \ldots, 6\}$.

\section{Numerical Analysis}

In this section, we study the numerical simulations of our model system on the basis of analytical findings. Our numerical studies were done using MATLAB 2008a. For numerical simulations we take a set of parameter values given in table (1). Most of the parameter values are taken from Segel (1984), Schulz (1994), Goudar et al. (1999), Kumar et al. (2012), Das and Arnold (2013), some are estimated and remaining values are assumed.

Table 1. Values of the parameters used in numerical simulation.

\begin{tabular}{|c|c|}
\hline Parameters & Values (Unit) \\
\hline$k_{1}$ & $(0.01-2.5)(\mu \mathrm{mol} / \mathrm{l})^{-1} h^{-1}$ \\
$k_{2}$ & $(0-3)(\mu \mathrm{mol} / \mathrm{l})^{-1} h^{-1}$ \\
$k_{3}$ & $(0-2.5)(\mu \mathrm{mol} / \mathrm{l})^{-1} h^{-1}$ \\
$k_{4}$ & $(0-3)(\mu \mathrm{mol} / \mathrm{l})^{-1} h^{-1}$ \\
$k_{-1}$ & $(0.001-0.3) h^{-1}$ \\
$k_{-2}$ & $(0-0.03) h^{-1}$ \\
$k_{-3}$ & $(0-0.09) h^{-1}$ \\
\hline
\end{tabular}

Figure 3 shows the reaction dynamics between protease inhibitors and cytochrome P3A4 (CYP3A4) enzyme in human liver in the absence of alcohol. While taking protease inhibitors $(I(t))$, the secretion of enzyme Cytochrome P3A4 $(E(t))$ occurs from the liver and it reacts with the drug forming CYP3A4-PI complex $\left(C_{1}(t)\right)$. So, the enzyme $(E(t))$ concentration and the PI $(I(t))$ concentration decrease and the complex concentration $\left(C_{1}(t)\right)$ increases with time in a particular rate. Further, a little portion of the complex $\left(C_{1}(t)\right)$ reverts back to the enzyme $(E(t))$ and PI $(I(t))$. So, the complex $\left(C_{1}(t)\right)$ concentration decreases and as the enzyme $(E(t))$ is reversed, the enzyme $(E(t))$ concentration slightly increases. But changing in the concentration of the drug PI $(I(t))$ is not noticeable because of its higher concentration. Although we can study the effect of reversion of the drug PI $(I(t))$ in other way. If there is no reversion of the PI $(I(t))$ from the complex $C_{1}$, the PI $(I(t))$ concentration will decrease more rapidly. But since the drug $(I(t))$ is reversed, the PI $(I(t))$ concentration decreases very slowly. 
International Journal of Mathematical, Engineering and Management Sciences

Vol. 4, No. 4, 922-935, 2019

https://dx.doi.org/10.33889/IJMEMS.2019.4.4-073
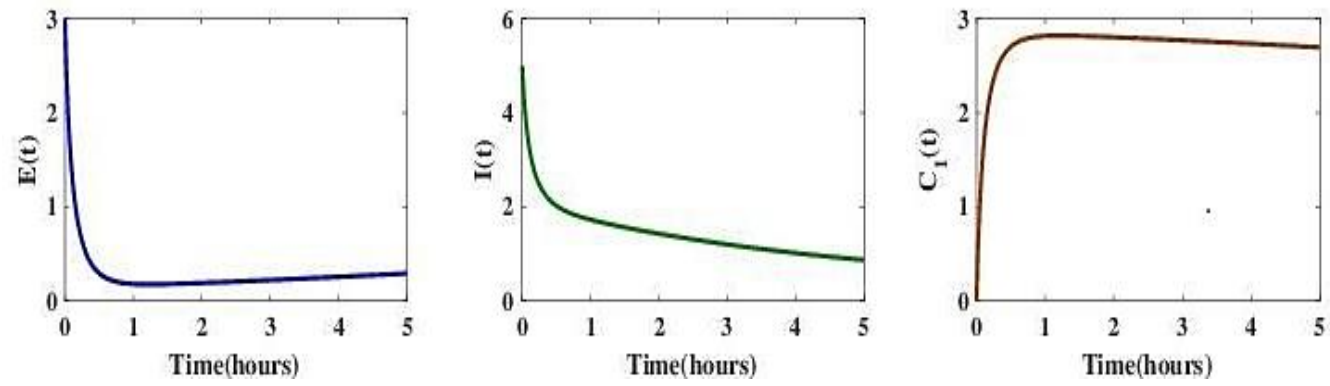

Figure 3. The nature of the curves of enzyme, protease inhibitor and complex $C_{1}$ without alcohol, using $E_{0}$ $=3.0 \mu \mathrm{mol} / \mathrm{L}, I_{0}=5.0 \mu \mathrm{mol} / \mathrm{L}, S_{0}=0 \mu \mathrm{mol} / \mathrm{L}$ and $C_{10}=0$ as initial values

Figure 4 describes the dynamics between protease inhibitors and cytochrome P3A4 enzyme in the liver after consumption of alcohol. When an HIV-infected individual consumes alcohol $(S(t))$, it goes to the liver and reacts with the liver secreted enzyme cytochrome P3A4 $(E(t))$. So, the concentration of alcohol $(S(t))$ and enzyme $(E(t))$ decreases and the complex $\left(C_{2}(t)\right)$ concentration increases with time at a particular rate. A little portion of the complex $\left(C_{2}(t)\right)$ reverts. Now the drug Protease Inhibitor $(I(t))$ reacts with the complex $\left(C_{2}(t)\right)$ forming a complex $\left(C_{3}(t)\right)$. For this, the complex $\left(C_{2}(t)\right)$ concentration decreases and as a little portion of the complex $\left(C_{3}(t)\right)$ reverts, the concentration of complex $\left(C_{2}(t)\right)$ slightly increases. Now, the complex $\left(C_{3}(t)\right)$ is converted into the product $(P(t))$ and the enzyme $(E(t))$ is reversed. So, the complex $\left(C_{3}(t)\right)$ concentration decreases when the product $(P(t))$ concentration increases and as the CYP3A4 enzyme $(E(t))$ is reversed, the enzyme $(E(t))$ concentration increases.
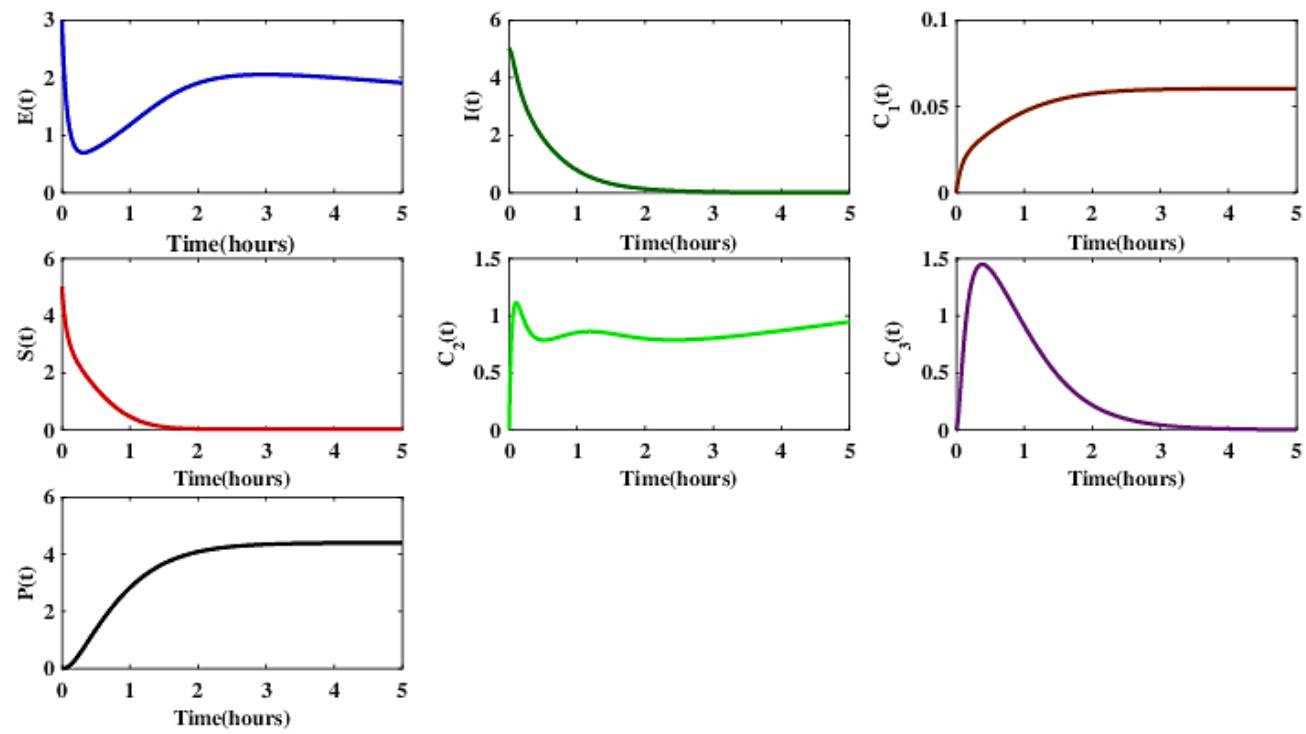

Figure 4. The nature of the curves of all reactants after alcohol consumption with the initial values $E_{0}=3.0$ $\mu \mathrm{mol} / \mathrm{L}, I_{0}=5.0 \mu \mathrm{mol} / \mathrm{L}, S_{0}=5.0 \mu \mathrm{mol} / \mathrm{L}$ and $C_{10}=0$ 
International Journal of Mathematical, Engineering and Management Sciences

Vol. 4, No. 4, 922-935, 2019

https://dx.doi.org/10.33889/IJMEMS.2019.4.4-073

In Figure 5, we compare the concentrations of enzyme CYP3A4, protease inhibitors and the complex $C_{1}$ in presence of alcohol and in the absence of alcohol. Here we observe that the formation of the complex $C_{1}$ slows down after consumption of alcohol. Because of the absorption of enzyme cytochrome P3A4 $(E(t))$ by alcohol as well as well as the reaction of the enzyme $(E(t))$ with the drug PI $(I(t))$, enzyme concentration decreases. But since the enzyme $(E(t))$ is reversed, the concentration of the enzyme $(E(t))$ increases. Also we can see that in presence of alcohol, the curve of protease inhibitor PI $(I(t))$ becomes lower than in absence of alcohol which is harmful for alcoholic HIV-infected individual. Until the complex $C_{2}$ is formed, PI reacts with the enzyme $(E(t))$ and produces the complex $C_{1}$. Hence, at first, in presence of alcohol the PI concentration slows down in a similar way that of the curve of PI in absence of alcohol. Another feature of the figure is that the curve of complex $C_{1}$ is like a horizontal curve in the presence of alcohol. Due to the presence of alcohol, the formation of complex $C_{1}$ is negligible and thus we see the impact of alcohol consumption on protease inhibitors for HIV-infected individuals.
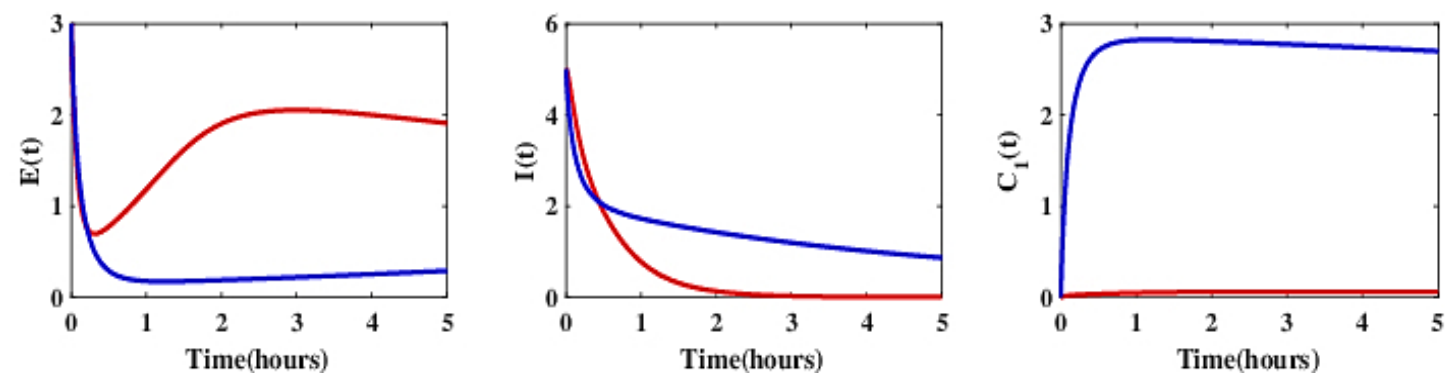

Figure 5. Comparison study between absence of alcohol and presence of alcohol for enzyme, protease inhibitor and complex $C_{1}$ with the initial conditions $E_{0}=3.0 \mu \mathrm{mol} / \mathrm{L}, I_{0}=5.0 \mu \mathrm{mol} / \mathrm{L}, S_{0}=$ $5.0 \mu \mathrm{mol} / L$ and $C_{10}=0$ [Blue line indicates absence of alcohol while red line indicates presence of alcohol]

\section{Conclusion}

In this paper, we mainly focus on the effect of alcohol consumption during antiretroviral therapy for HIV-infected individuals. The PIs used in the HAART regimen interact with alcohol thereby altering their metabolism and antiviral activity. Here we show that Alcohol and PIs interact with CYP3A4 physically causing complex alcoholCYP3A4-PI, which results in altered metabolism of PI as well as NNRTI in the liver. In this article, we have constructed a mathematical model using enzyme kinetic reaction, which seems to be rare in HIV disease dynamics. We can see from the Figure 5 that for the consumption of alcohol, the PI concentration has been reduced. Thus alcohol prevents the inhibition property of PI towards enzyme CYP3A4 and increases the metabolism rate of PI by CYP3A4. Conclusively, we expect that our work would redirect the mathematicians to explore new treatment strategy that effectively treats alcoholic HIV+ patients in a more balanced way. 
International Journal of Mathematical, Engineering and Management Sciences

Vol. 4, No. 4, 922-935, 2019

https://dx.doi.org/10.33889/IJMEMS.2019.4.4-073

\section{Conflict of Interest}

The authors confirm that there are no known conflicts of interest associated with this publication.

\section{Acknowledgement}

Srijita Mondal and Priyanka Ghosh are the recipient of "Innovation in Science Pursuit for Inspired Research" (INSPIRE) Program Fellowship, Department of Science and Technology, Government of India.

\section{References}

Acosta, E.P. (2002). Pharmacokinetic enhancement of protease inhibitors. Journal of Acquired Immune Deficiency Syndromes (1999), 29, S11-8.

Biswas, D., Datta, A., \& Roy, P.K. (2016). Combating Leishmaniasis through awareness campaigning: a mathematical study on media efficiency. International Journal of Mathematical, Engineering and Management Sciences, 1(3), 139-149.

Chander, G. (2011). Addressing alcohol use in HIV-infected persons. Topics in Antiviral Medicine, 19(4), 143-147.

Chander, G., Himelhoch, S., Fleishman, J.A., Hellinger, J., Gaist, P., Moore, R.D., \& Gebo, K.A. (2009). HAART receipt and viral suppression among HIV-infected patients with co-occurring mental illness and illicit drug use. AIDS Care, 21(5), 655-663.

Chander, G., Lau, B., \& Moore, R.D. (2006). Hazardous alcohol use: a risk factor for non-adherence and lack of suppression in HIV infection. Journal of Acquired Immune Deficiency Syndromes (1999), 43(4), 411-417.

Chatterjee, A.N., \& Roy, P.K. (2012). Anti-viral drug treatment along with immune activator IL-2: a control-based mathematical approach for HIV infection. International Journal of Control, 85(2), 220237.

Chowdhury, S., \& Roy, P.K. (2016). Mathematical modelling of enfuvirtide and protease inhibitors as combination therapy for HIV. International Journal of Nonlinear Sciences and Numerical Simulation, 17(6), 259-275.

Das, K., \& Arnold, E. (2013). HIV-1 reverse transcriptase and antiviral drug resistance. Part 1. Current Opinion in Virology, 3(2), 111-118.

Ferrari, L.F., \& Levine, J.D. (2010). Alcohol consumption enhances antiretroviral painful peripheral neuropathy by mitochondrial mechanisms. European Journal of Neuroscience, 32(5), 811-818.

Flexner, C.W., Cargill, V.A., Sinclair, J., Kresina, T.F., \& Cheever, L. (2001). Alcohol use can result in enhanced drug metabolism in HIV pharmacotherapy. AIDS Patient Care and STDs, 15(2), 57-58.

Ghosh, P., \& Roy, P.K. (2018). Mathematical modelling of methanol poisoning with impulsive dosing of antidote therapeutics to prevent toxicity. Mathematical Methods in the Applied Sciences, 41(18), 91769190.

Ghosh, P., Cao, X., Pal, J., Chowdhury, S., Saha, S., Nandi, S., \& Roy, P.K. (2018). Mathematical modeling for the prevention of methanol poisoning through ethanol by impulsive way. Differential Equations and Dynamical Systems, 1-18.

Gonzalez, A., Barinas, J., \& O’Cleirigh, C. (2011). Substance use: impact on adherence and HIV medical treatment. Current HIV/AIDS Reports, 8(4), 223-234. 
International Journal of Mathematical, Engineering and Management Sciences

Vol. 4, No. 4, 922-935, 2019

https://dx.doi.org/10.33889/IJMEMS.2019.4.4-073

Goudar, C.T., Sonnad, J.R., \& Duggleby, R.G. (1999). Parameter estimation using a direct solution of the integrated Michaelis-Menten equation. Biochimica et Biophysica Acta (BBA)-Protein Structure and Molecular Enzymology, 1429(2), 377-383.

Hendershot, C.S., Stoner, S.A., Pantalone, D.W., \& Simoni, J.M. (2009). Alcohol use and antiretroviral adherence: review and meta-analysis. Journal of Acquired Immune Deficiency Syndromes (1999), 52(2), 180-202.

Hirsch, M.S., Günthard, H.F., Schapiro, J.M., Vézinet, F.B., Clotet, B., Hammer, S.M., Johnson, V.A., Kuritzkes, D.R., Mellors, J.W., Pillay, D., Yeni, P.G, Jacobsen, D.M., \& Richman, D.D. (2008). Antiretroviral drug resistance testing in adult HIV-1 infection: 2008 recommendations of an International AIDS Society-USA panel. Clinical Infectious Diseases, 47(2), 266-285.

Kostyuchenko, Y.V. (2018). On the methodology of satellite data utilization in multi-modeling approach for socio-ecological risks assessment tasks: a problem formulation. International Journal of Mathematical, Engineering and Management Sciences, 3(1), 1-8.

Kumar, S., \& Kumar, A. (2011). Differential effects of ethanol on spectral binding and inhibition of cytochrome P450 3A4 with eight protease inhibitors antiretroviral drugs. Alcoholism: Clinical and Experimental Research, 35(12), 2121-2127.

Kumar, S., Jin, M., Ande, A., Sinha, N., Silverstein, P.S., \& Kumar, A. (2012). Alcohol consumption effect on antiretroviral therapy and HIV-1 pathogenesis: role of cytochrome P450 isozymes. Expert Opinion on Drug Metabolism \& Toxicology, 8(11), 1363-1375.

Lou, J., \& Smith, R.J. (2011). Modelling the effects of adherence to the HIV fusion inhibitor enfuvirtide. Journal of Theoretical Biology, 268(1), 1-13.

Midde, N.M., Rahman, M.A., Rathi, C., Li, J., Meibohm, B., Li, W., \& Kumar, S. (2016). Effect of ethanol on the metabolic characteristics of HIV-1 integrase inhibitor elvitegravir and elvitegravir/cobicistat with CYP3A: An analysis using a newly developed LC-MS/MS method. PloS one, 11(2), e0149225.

Miguez, M.J., Shorposner, G.A.I.L., Morales, G., Rodriguez, A., \& Burbano, X. (2003). HIV treatment in drug abusers: impact of alcohol use. Addiction Biology, 8(1), 33-37.

Norman, L.R., \& Kumar, A. (2006). Neuropscyhological complications of HIV disease and substances of abuse. American Journal of Infectious Diseases, 2(2), 67-73.

Pal, D., \& Mitra, A.K. (2006). MDR-and CYP3A4-mediated drug-drug interactions. Journal of Neuroimmune Pharmacology, 1(3), 323-339.

Purohit, V., Rapaka, R., \& Shurtleff, D. (2011). Drugs of abuse, dopamine, and HIV-associated neurocognitive disorders/HIV-associated dementia. Molecular Neurobiology, 44(1), 102-110.

Roy, P.K., Saha, S., \& Al Basir, F. (2015). Effect of awareness programs in controlling the disease HIV/AIDS: an optimal control theoretic approach. Advances in Difference Equations, 2015(1), 217234.

Schulz, A.R. (1994). Enzyme kinetics: from diastase to multi-enzyme systems. Cambridge University Press.

Segel, L.A. (1984). Modeling dynamic phenomena in molecular and cellular biology. Cambridge University Press.

Smith, R.J. (2008). Explicitly accounting for antiretroviral drug uptake in theoretical HIV models predicts long-term failure of protease-only therapy. Journal of Theoretical Biology, 251(2), 227-237.

Smith, R.J., \& Wahl, L.M. (2004). Distinct effects of protease and reverse transcriptase inhibition in an immunological model of HIV-1 infection with impulsive drug effects. Bulletin of Mathematical Biology, 66(5), 1259-1283. 
International Journal of Mathematical, Engineering and Management Sciences

Vol. 4, No. 4, 922-935, 2019

https://dx.doi.org/10.33889/IJMEMS.2019.4.4-073

Smith, R.J., \& Wahl, L.M. (2005). Drug resistance in an immunological model of HIV-1 infection with impulsive drug effects. Bulletin of Mathematical Biology, 67(4), 783-813.

Song, B.J., Lou, J., \& Wen, Q.Z. (2011). Modelling two different therapy strategies for drug T-20 on HIV1 patients. Applied Mathematics and Mechanics, 32(4), 419-436.

Walubo, A. (2007). The role of cytochrome P450 in antiretroviral drug interactions. Expert Opinion on Drug Metabolism \& Toxicology, 3(4), 583-598.

Witteveen, E., \& van Ameijden, E.J. (2002). Drug users and HIV-combination therapy (HAART): factors which impede or facilitate adherence. Substance Use \& Misuse, 37(14), 1905-1925.

Xu, L., \& Desai, M.C. (2009). Pharmacokinetic enhancers for HIV drugs. Current opinion in investigational drugs (London, England: 2000), 10(8), 775-786. 